\title{
Condições higiênicas e sanitárias de unidades de alimentação e nutrição de escolas em tempo integral
}

\author{
Hygienic and sanitary conditions in food services of full-time schools \\ Condiciones higiénicas y sanitarias de unidades de alimentación y nutrición de escuelas \\ en tiempo integral
}

Thaís Ingrid Leal Mendes ${ }^{1 *}$, Marina Luiza Santos Monteiro ${ }^{1}$, Luiza Marly Freitas de Carvalho ${ }^{1}$, Keila Cristiane Batista Bezerra ${ }^{1}$.

\section{RESUMO}

Objetivo: Avaliar as estruturas físicas e as condições higiênico-sanitárias quanto à produção de refeições em unidades de alimentação e nutrição de escolas integrais. Método: Trata-se de estudo transversal, de caráter quantitativo e descritivo, onde foram analisadas a estrutura física e aferição das temperaturas de preparações servidas em 2 escolas de tempo integral. Os dados foram analisados com auxílio do software IBM Statistical. Resultados: A avaliação por itens permitiu evidenciar que o maior percentual de inconformidade foi verificado nos itens documentação e registros e equipamento de proteção individual, sendo encontrado 100\% e 16,7\%, respectivamente, na unidade de alimentação e nutrição I e, na unidade de alimentação e nutrição II, $100 \% \mathrm{e}$ $50 \%$, respectivamente. Dentre os itens, os de edificações e instalações, produção e transporte do alimento apresentaram maior índice de conformidade com relação as duas UAN's. Quanto à avaliação da média de temperatura, as preparações servidas quentes, em alguns momentos, apresentaram resultados abaixo do padrão recomendado,, o que se relaciona à ausência de balcão térmico em ambas as unidades, contribuindo para a variação desses valores. Conclusão: A correção das inadequações encontradas no estudo se faz necessária para garantir a qualidade e segurança das preparações oferecidas nas unidades.

Palavras-chave: Refeições, Alimentação, Escolas.

\begin{abstract}
Objective: To evaluate the physical structures and hygienic-sanitary conditions regarding the production of meals in units of feeding and nutrition in integral schools. Methods: It is a cross-sectional, quantitative and descriptive study, where the physical structure and temperature gauging of prepared meals were analyzed in 2 full-time schools. The data were analyzed using the IBM Statistical software. Results: The evaluation by items allowed to show that the highest percentage of nonconformity was verified in the items documentation and records and personal protection equipment, being found the percentage of $100 \%$ and $16.7 \%$, respectively, in food services I and, in food services II, $100 \%$ and 50\%, respectively. Among the items, those of buildings and facilities and production and transportation of food had a higher rate of compliance with respect to two UAN's. As for the evaluation of the average temperature, the hot prepared preparations, in some moments, presented results below the recommended standard, which is related to the absence of thermal counter in both units, contributing to the variation of these values. Conclusion: Correction of the inadequacies found in the study is necessary to guarantee the quality and safety of the preparations offered at the units.
\end{abstract}

Key words: Meals, Food, Schools.

${ }^{1}$ Centro Universitário Santo Agostinho, Teresina-PI. *E-mail: thaisingridmendes@gmail.com

SUBMETIDO EM: 6/2019

ACEITO EM: 7/2019

PUBLICADO EM: 8/2019

REAS/EJCH | Vol.Sup.31 | e1150 | DOI: https://doi.org/10.25248/reas.e1150.2019 Página 1 de 10 


\section{RESUMEN}

Objetivo: Evaluar las estructuras físicas y las condiciones higiénico-sanitarias en cuanto a la producción de comidas en unidades de alimentación y nutrición de escuelas integrales. Métodos: Se trata de un estudio transversal, de carácter cuantitativo y descriptivo, donde se analizaron la estructura física y la medición de las temperaturas de preparaciones servidas en 2 escuelas de tiempo completo. Los datos fueron analizados con ayuda del software IBM Statistical. Resultados: La evaluación por ítems permitió evidenciar que el mayor porcentaje de inconformidad fue verificado en los ítems documentación y registros y equipo de protección individual, siendo encontrado $100 \%$ y el $16,7 \%$, respectivamente, en la unidade de alimentación y nutrición I y, en la unidade de alimentación y nutrición II, $100 \%$ y 50\%, respectivamente. Entre los ítems, los de edificaciones e instalaciones y producción y transporte del alimento presentaron mayor índice de conformidad con relación a dos UAN's. En cuanto a la evaluación de la media de temperatura, las preparaciones servidas calientes, en algunos momentos, presentaron resultados por debajo del padrón recomendado, lo que se relaciona con la ausencia de balcón térmico en ambas unidades, contribuyendo a la variación de estos valores. Conclusión: La corrección de las inadecuaciones encontradas en el estudio se hace necesaria para garantizar la calidad y seguridad de los preparados ofrecidos en las unidades.

Palabras clave: Comidas, Alimentación, Escuelas.

\section{INTRODUÇÃO}

As Unidades de Alimentação e Nutrição (UAN) são serviços produtores de alimentação para coletividades que possuem um conjunto de áreas definidas, organizadas em sequências, e que fornecem refeições balanceadas. A UAN deve possuir além dos padrões dietéticos, os higiênicos, ajustados às condições financeiras do local, no intuito final de atender às necessidades nutricionais de seus comensais (BARBOSA ILS, 2017).

Segundo Pohren NF, et al. (2014), estas possuem estrutura organizacional simples, mas se tornam complexas, dependendo do tipo e quantidade de refeições produzidas e do tipo de gerenciamento e contrato. Pertencem ao setor de alimentação coletiva e possuem um compromisso com a saúde, pois oferecem aos comensais uma alimentação equilibrada, respeitando as Leis da Alimentação, como a quantidade, qualidade, harmonia e adequação. Trasel J e Junior SBJ (2014), apontam em estudos que o armazenamento nas preparações em temperaturas inadequadas e as más condições de higiene do local são as principais causas das ocorrências de Doenças Transmitidas por Alimentos (DTA).

Gomes MFFA, et al. (2015), afirmam que o serviço de alimentação escolar tem a missão de complementar as necessidades nutricionais dos alunos através da alimentação produzida e distribuída diariamente. $O$ fornecimento de refeições, em instituições públicas de ensino envolve um conjunto de ferramentas para a garantia da qualidade e segurança, tendo como finalidade promover, manter ou mesmo recuperar a saúde individual e coletiva dos usuários que se beneficiam da alimentação servida.

Para Pederssetti MT e Hautrive TP (2016), de acordo com estudos estatísticos da Organização Mundial de Saúde (OMS), mais de 60\% dos casos de doenças de origem alimentar decorrem do descuido higiênicosanitário de manipuladores, das técnicas inadequadas de processamento e da deficiência de higiene da estrutura física, utensílios e equipamentos. Gomes NAAA, et al. (2012), afirmam a qualidade higiênicosanitária como fator de segurança alimentar tem sido muito estudada e discutida, uma vez que a incidência de DTA vem aumentando em nível mundial.

De acordo com Medeiros MGGA, et al. (2017), a realização de boas práticas de manipulação dos alimentos previne a ocorrência de problemas relacionados à higiene, como o crescimento de micro-organismos nas preparações que prejudicam a saúde dos comensais.

Conforme Ribeiro ESS (2017), para garantir a implementação de um controle higiênico-sanitário eficaz, as UAN's devem cumprir as leis estabelecidas pela Agência Nacional de Vigilância Sanitária (ANVISA). As 
especificações desta legislação tratam sobre alguns pontos como as edificações, instalações, móveis e utensílios, abastecimento de água, abastecimento de gás, iluminação, teto, paredes e pisos, higiene sanitária das edificações, móveis e utensílios, higiene dos manipuladores de alimentos bem como, registros, documentos e responsabilidade.

Desta forma, este estudo teve como objetivo avaliar as estruturas físicas e as condições higiênico-sanitárias quanto à produção de refeições em unidades de alimentação e nutrição de escolas de tempo integral.

\section{MÉTODOS}

Trata-se de estudo de delineamento transversal, de caráter quantitativo e descritivo, sendo realizada a pesquisa no período de novembro de 2018 a março de 2019. Participaram do estudo 2 unidades de alimentação e nutrição escolar de tempo integral, onde foram analisadas a estrutura física e aferição das temperaturas servidas em cada preparação, executado no município de Teresina-PI. Estas unidades foram identificadas como UAN I e UAN II para comparação e análise das características observadas. A UAN I produz, em média, 350 refeições por dia; já a UAN II produz, em média, 200 refeições, com maior número de refeições sendo servidas no almoço.

Para a avaliação das estruturas físicas e das condições higiênico-sanitárias, foi aplicado um formulário nas 2 instituições o Checklist - Lista de Verificação das Condições Higiênico-Sanitárias baseada na RDC nํ216/2004 e 275/2002, o referido documento permite observar e analisar os seguintes itens: edificação e instalações composto por ( $n=64$ itens); produção e transporte do alimento com ( $n=32$ itens); documentação e registro ( $n=7$ itens); equipamento de proteção individual ( $n=6$ itens), totalizando 109 itens inerentes à Checklist.

Para caracterização das conformidades e não conformidades, utilizaram-se os termos "Sim" e "Não", respectivamente, em cada item avaliado, assinalando um "x" nas colunas da Checklist. Quando marcado "Sim", o item atendeu à conformidade do local em boas práticas e, quando marcado "Não", o item não atendeu à legislação. Além das colunas "Sim" e "Não", havia ainda a opção de marcar um "x" na coluna "NA" (Não se Aplica), em caso de o critério não condizer com o tipo da UAN avaliada.

Em seguida, a aferição das temperaturas de cada preparação foi realizada durante o início da sua distribuição, sendo elas a colação, almoço e lanche da tarde, observando se está de acordo como determina a Agência Nacional de Vigilância Sanitária - ANVISA. A verificação da temperatura das preparações foi analisada com o Termômetro GM-300 Laser Digital Infravermelho com variação -50 a $420 \stackrel{\circ}{\circ}$. As aferições das temperaturas ocorreram durante cinco dias da semana em cada uma das UAN's. Após a coleta, os dados foram armazenados e tabulados em planilha eletrônica Microsoft Office Excel e analisados no programa IBM Statistical Package for the Social Sciences versão 20.0, sendo colocados em percentuais.

\section{RESULTADOS E DISCUSSÃO}

Participaram da pesquisa 2 escolas de tempo integral de ensino, foi aplicado um formulário o Checklist Lista de Verificação das Condições Higiênico-Sanitárias de acordo com a resolução RDC no 216/04 e 275/02, apresentando-se a quantidade de itens conforme e não conforme colocados, com total de 109 itens avaliados.

Em relação ao item edificações e instalações, onde foram relacionadas às características físicas e estruturais como piso, teto, paredes e divisórias, janelas, lavatórios, iluminação, esgotamento sanitário, verificou-se percentual de conformidade de $90,6 \%(n=58)$, apresentando fluxo ordenado sem possibilidades de cruzamento nas etapas de preparação dos alimentos, e 9,4\% $(n=6)$ não conforme, com relação à inexistência de proteção contra insetos e roedores, a falta de lixeira com acionamento por pedal, podendo comprometer a qualidade final das preparações (Gráfico 1).

Em um estudo realizado por Lopes ACC et al. (2015), identificaram resultado parecidos ao encontrado neste presente estudo ao observarem que, em algumas unidades escolares, não era realizado com frequência o controle de insetos e pragas urbanas, mas também havia a ausência de medidas preventivas importantes, como ausência de telas nas janelas e teto descoberto, além da presença de insetos e teias de aranha. 
Gráfico 1 - Percentual da conformidade e não conformidade do Cheklist da UAN I. Teresina-PI, 2018.

120

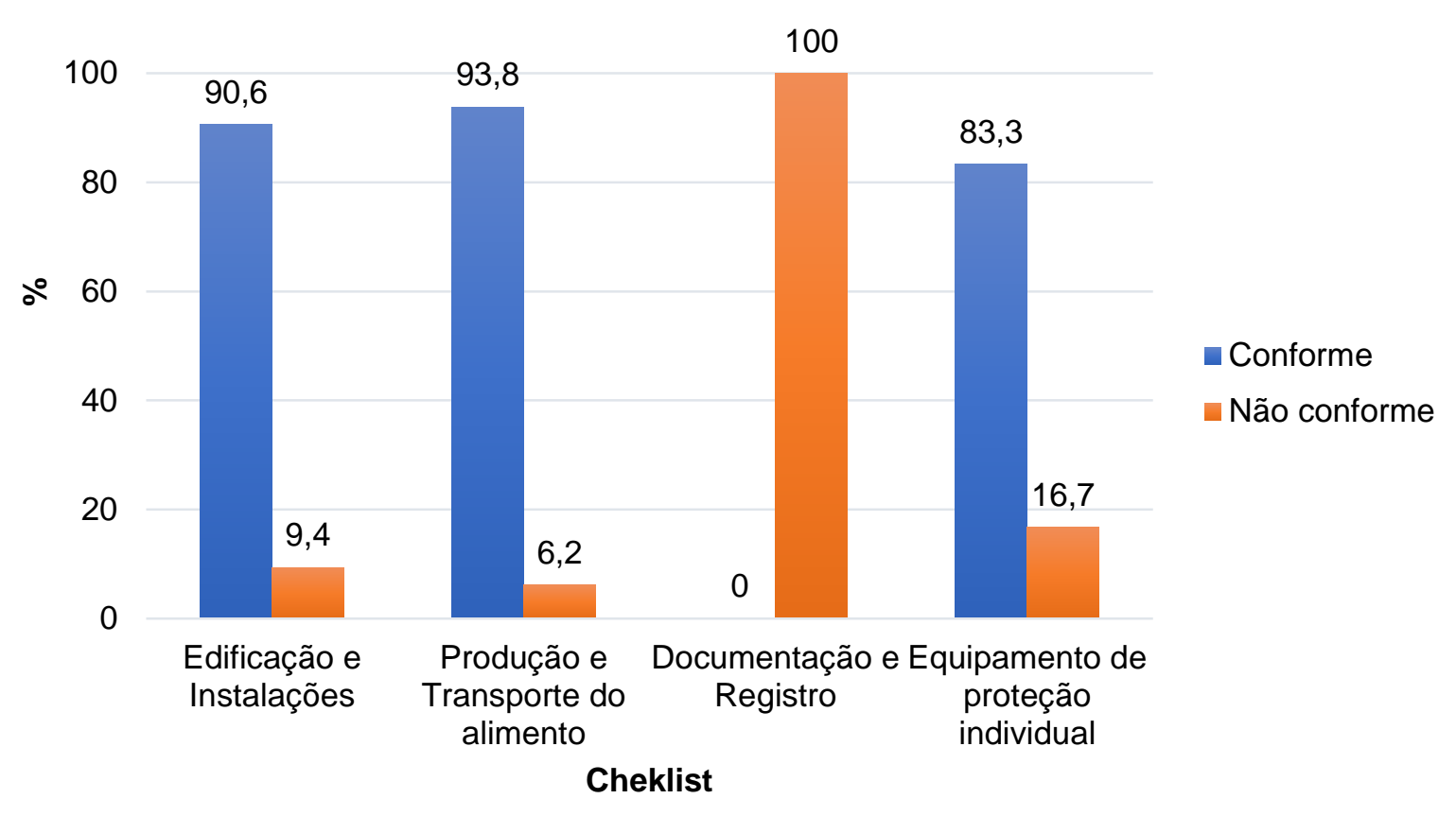

Fonte: Dados da pesquisa, 2018.

Para o item de produção e transporte do alimento, este bloco apresentou $93,8 \%(n=30)$ de conformidade, destacando-se o uso da matéria-prima, ingredientes e embalagens, respeitando o prazo de validade da mesma. Com relação ao item não conforme, com $6,2 \%(n=2)$, destaca-se a ausência de área isolada e protegida para a recepção da matéria-prima, ingredientes e embalagens, bem como alimentos colocados diretamente no chão, permitindo, assim, a proliferação de pragas e roedores como também a diminuição da vida de prateleira da mesma. Em relação à documentação e registro, foi encontrado $0 \%(n=0)$ de conformidade e $100 \%$ ( $n=7)$ de não conformidade, observando a ausência do Manual de Boas Práticas (MBP), bem como dos Procedimentos Operacionais Padronizados (POP's), ficando evidente que os manipuladores não tinham acesso nem o hábito de consultá-los. De acordo com a RDC 216/04, os serviços de alimentação devem implementar os procedimentos de boas práticas, proporcionando a garantia das condições higiênicosanitárias dos alimentos manipulados. As POP's vêm com o propósito de agregar esses conhecimentos aos manipuladores sobre boas práticas (Gráfico 1).

Silva LM (2016), obtiveram resultados que divergem com o encontrado no referido estudo, pois verificaram que as instituições analisadas possuíam uma maior adequação, visto que todas as escolas possuíam MBP e POP, porém esses documentos não estavam disponíveis aos colaboradores e órgãos fiscalizadores, e ainda muitos diretores e manipuladores de alimentos desconheciam seu conteúdo.

Com relação ao item de equipamentos de proteção individual, apresentou $83,3 \%(n=5)$ de conformidade, sendo todos paramentados e uniformizados, e apenas $16,7 \%(n=1)$ da amostra de não conformidade, visto que os calçados utilizados não eram adequados e não estavam em bom uso (alguns manipuladores relatavam sentir incômodos ao usá-los) e, em relação às luvas, os manipuladores não tinham o hábito de usar para preparar as refeições, somente eram utilizadas para o preparo da salada, sendo este EPI importante para evitar o contato da superfície da mão diretamente com o alimento (Gráfico 1).

Melo VL et al. (2014), ao avaliarem uma UAN de Restaurante Universitário, verificaram que os manipuladores atuantes diretamente na produção de alimentos utilizavam filme de PVC enrolado nas pernas juntamente com as botas. Segundo os trabalhadores, isso evita o desconforto de trabalhar com a bota molhada e entrar em atrito com a perna da calça do uniforme. 
Os percentuais avaliados na UAN II permitiram evidenciar que o item de edificações e instalações apresentou $90,6 \%(n=58)$ de conformidade em relação ao teto, piso, paredes e divisórias, climatização e esgotamento sanitário. Com relação ao item de não conformidade, apresentou apenas $9,4 \%(n=6)$ com ausência de lixeiras com acionamento de pedal, ausência de porta com acionamento automático e proteção contra insetos e roedores (Gráfico 2).

Gráfico 2 - Percentual da conformidade e não conformidade do Cheklist da UAN II. Teresina-PI, 2018.

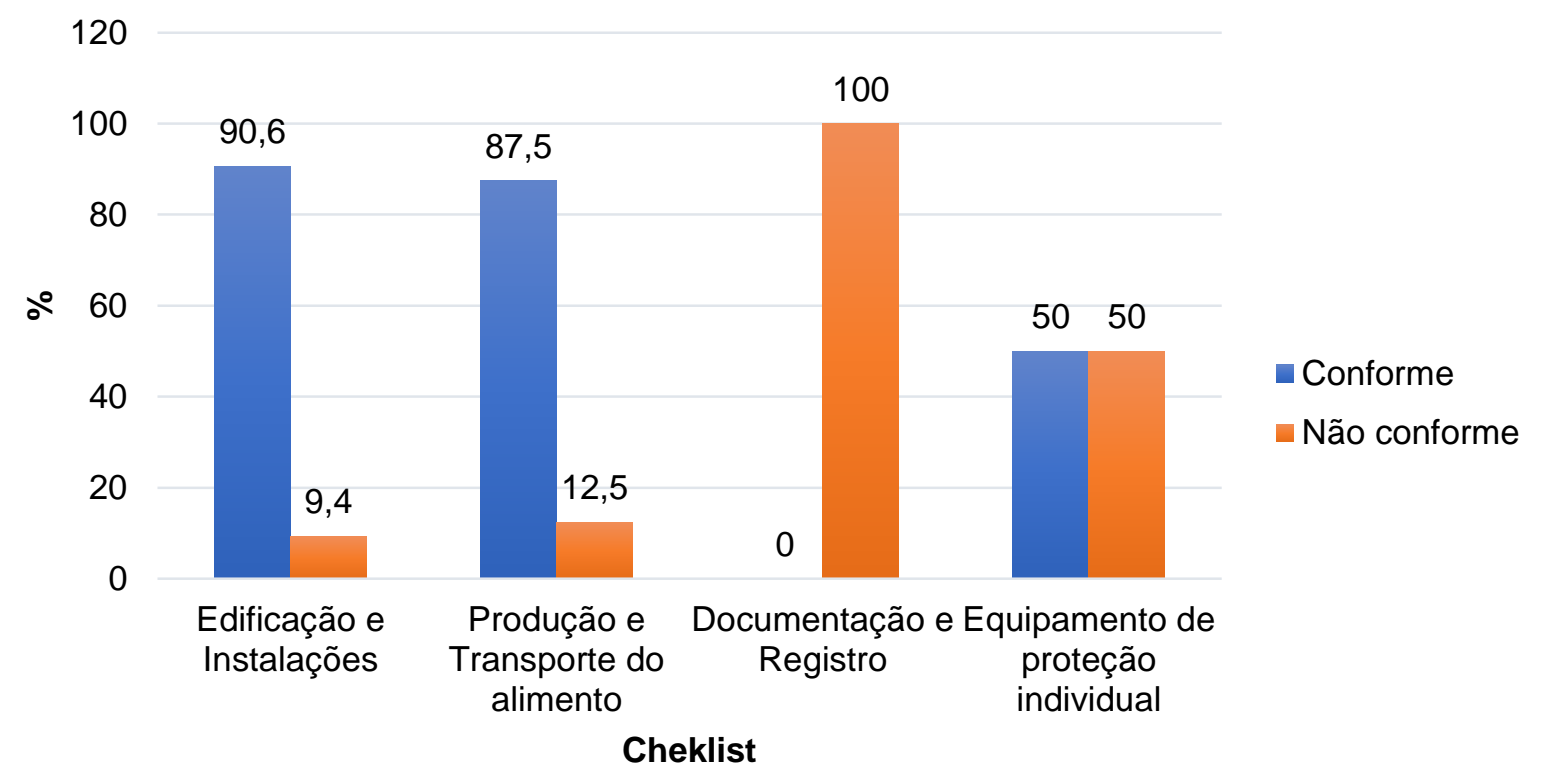

Fonte: Dados da pesquisa, 2018.

Rasquinha BS et al. (2017), em seu estudo não obtiveram maior percentual de conformidade na categoria de edificações e instalações em decorrência da falta de separações por meios físicos que evitam a contaminação cruzada entre as atividades de pré-preparo, preparo e higienização, sendo feitas no mesmo local e ao mesmo tempo. Este método facilita a contaminação pelo contato entre alimentos crus e cozidos e até mesmo com os produtos saneantes utilizados na higienização. O item de produção e transporte do alimento apresentou $87,5 \%$ ( $n=28)$ de conformidade de acordo com o transporte e qualidade do produto final, sendo $12,5 \%(n=4)$ de itens não conforme e inadequado o controle da circulação e acesso do pessoal (Gráfico 2).

De acordo com Ramos AE et al. (2015), seu estudo apresentou 100\% de conformidade com relação ao bloco de produção e transporte do alimento exigidos pela legislação, tendo todo o cuidado com a matéria-prima, o fluxo de produção, armazenagem e transporte do produto final. No item documentação e registro, obtiveram $0 \%(n=0)$ de conformidade e 100\% ( $n=7)$ de não conformidade, sendo observada na UAN's escolar a ausência do Manual de Boas Práticas (MBP) e dos POP's - Procedimentos Operacionais Padronizados. Não havia o cumprimento dos POP's, porém na unidade estava estabelecida a sua importância. Este documento descreve de forma clara e prática as operações rotineiras específicas, contribuindo para a garantia das refeições e a promoção das boas práticas na Unidade de Alimentação e Nutrição (Gráfico 2).

No trabalho de Nunes CNM et al. (2014), verificaram que, na unidade de alimentação e nutrição que analisavam, apenas $9 \%$ não seguiam os procedimentos realizados preconizados pelos POP's. No item de equipamento de proteção individual houve igualdade com relação ao percentual, sendo apresentados $50 \%$ ( $n=3$ ) de itens conforme e $50 \%(n=3)$ de itens não conforme, havendo diferença significativa quando comparada a UAN I. Verificou-se que os manipuladores utilizavam toucas, aventais e que tinham um lugar próprio para a guarda desses pertences, mas seu uso não era obrigatório e os uniformes e calçados não estavam em bom estado de conservação. Além disso, os manipuladores não utilizavam luvas e calças, relatando sentir incômodo ao serem questionados sobre o não uso desses equipamentos (Gráfico 2). 
Em um estudo no qual foram analisadas oito unidades produtoras de refeições, constatou-se que havia negligência em relação ao uso dos EPI's durantes as atividades, em especial o avental térmico que apresentou $90 \%$ de inadequação em nove unidades, podendo causar riscos aos colaboradores. Já na maior unidade estudada, observou-se descuido em relação à área de preparação dos alimentos, principalmente no que se refere ao uso da luva de aço (81,5\%) (ZANETIN PM e FATEL ECS, 2017).

Os percentuais de conformidade e não conformidade das Unidades de Alimentação e Nutrição Escolar conforme os itens avaliados foram obtidos por meio do percentual de dois formulários (Checklist) aplicados em unidades escolares de tempo integral em períodos distintos. Podemos constatar que a UAN I, ao ser avaliada no segundo semestre de 2018, apresentou maior percentual de conformidade com $85,3 \%$ ( $n=93$ ), classificado como muito bom. Já a UAN II, avaliada também no segundo semestre de 2018 , obteve média de adequação $81,7 \%$ $(n=89)$, recebendo a mesma classificação anterior da UAN I (Gráfico 3).

Gráfico 3 - Percentual de comparação da conformidade e não conformidade geral do Cheklist das duas UAN's escolares. Teresina-PI, 2018.

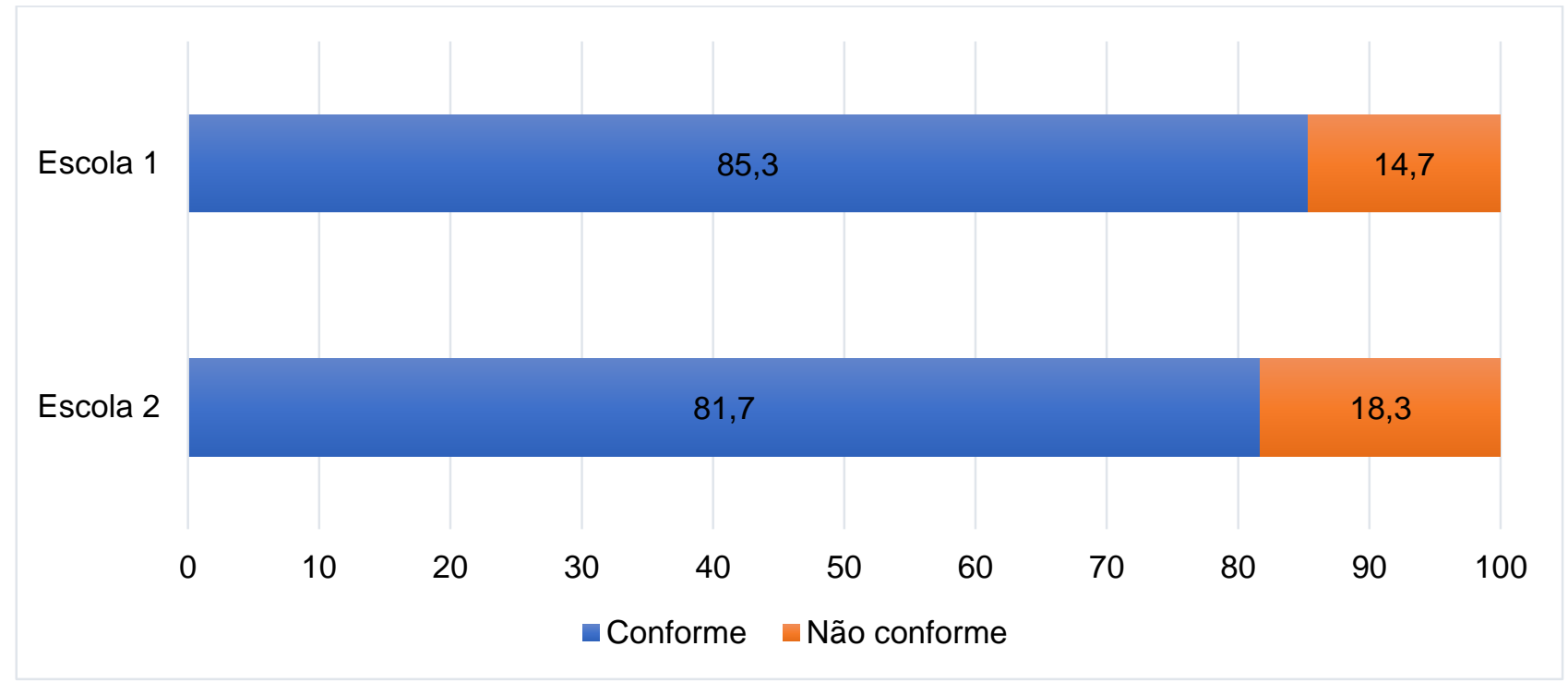

Fonte: Dados da pesquisa, 2018.

Resultado de estudo semelhante a este foi encontrado ao analisar o índice de adequação das UAN's de oito escolas de Caxias-MA, onde observaram que apenas duas UAN's $C$ e $D$ foram as únicas classificadas como grau 1 (70 a 100\%) de itens conforme, enquanto as UAN's B, G e H foram classificadas como grau 2 (51 a 69\%), e as UAN's A, E F foram categorizadas com grau 3 (0 a 50\%) (GOMES RNS, et al., 2016).

Devido ao grau de insatisfação em ambas as unidades estudadas, pode-se observar o índice em relação a não conformidade, tendo como principal fator contribuinte o não uso de EPI's adequado ao manipular os alimentos, bem como o desconhecimento dos POP's e sua utilização dentro da UAN (Gráfico 3).

Os manipuladores de alimentos devem receber treinados de maneira periódica enfatizando as Boas Práticas de Fabricação, visando aprimorar suas práticas corretas de higienização, fazendo com que a unidade de alimentação seja um local seguro, livre de contaminação para se alimentar, além de adquirir conhecimentos sobre organização do ambiente e desenvolvimento de habilidades (NETO AB, et al., 2015).

Com relação à média das temperaturas ao início da distribuição durante os cinco dias da semana referentes à avaliação das preparações da colação, verificou-se que ambas estiveram dentro do padrão determinado pela portaria CVS 5/2013. Em relação às preparações de temperatura ambiente, houve variação em relação à média do valor encontrado para o recomendado tanto no almoço como no lanche da tarde, devido à ausência de acondicionamento da UAN, podendo ocorrer à proliferação de micro-organismos, colocando em risco à saúde dos alunos (Tabela 1). 
Tabela 1 - Média das temperaturas encontradas nas preparações no início da distribuição da UAN I. TeresinaPI, 2019.

\begin{tabular}{lcccc}
\hline \hline \multicolumn{1}{c}{ Refeições } & $\begin{array}{c}\text { Média Encontrada } \\
\left({ }^{\circ} \mathbf{C}\right)\end{array}$ & $\begin{array}{c}\text { Valor Mínimo } \\
(-\mathbf{O})\end{array}$ & $\begin{array}{c}\text { Valor Máximo } \\
\left({ }^{\circ} \mathbf{C}\right)\end{array}$ & $\begin{array}{c}\text { Recomendada } \\
\left({ }^{\circ} \mathbf{C}\right)\end{array}$ \\
\hline COLAÇÃO & & & & \\
Preparações Quentes & 79,3 & 26 & 62,4 & $\uparrow 60-65$ \\
Preparações Frias & 3,5 & 8 & 9,6 & Até 10 \\
Preparações Ambientes & 10 & 24,6 & 25,5 & $10-21$ \\
ALMOÇO & & & & \\
Preparações Quentes & 132,9 & 45,2 & 67 & $\uparrow 60-65$ \\
Preparações Frias & - & - & - & Até 10 \\
Preparações Ambientes & 38,2 & 23,5 & 31,5 & $10-21$ \\
& & & & \\
LANCHE DA TARDE & - & - & - & Até 10 \\
Preparações Quentes & 8,5 & 7,8 & 17 & $10-21$ \\
Preparações Frias & 28,9 & 26,8 & 30,2 & \\
Preparações Ambientes & & &
\end{tabular}

Fonte: Dados da pesquisa, 2019.

Para garantir temperaturas adequadas na distribuição de refeições, é necessário que os equipamentos utilizados na distribuição sejam rigorosamente monitorados quanto ao controle de temperatura (WIETHOLTER MJ e FASSINA P, 2017). O bolo de goma servido na colação como preparação quente, obteve temperatura de $26 \stackrel{\circ}{\circ}$ em seu valor mínimo visto que o recomendado pela ANVISA e CVS 5/13 está entre 60-65 ㄷ. Essa variação de temperatura ocorreu visto que o bolo era mantido exposto até ser entregue aos alunos. O leite servido na colação obteve temperatura de $62,4{ }^{\circ} \mathrm{C}$ em seu valor máximo, estando dentro dos padrões recomendados. Nas preparações frias os sucos obtiveram temperaturas de $8{ }^{\circ} \mathrm{C}$ e $9,6^{\circ} \mathrm{C}$ estando dentro do recomendado, visto que 0 ideal para preparações frias é de até $10^{\circ} \mathrm{C}$. As preparações que eram mantidas em temperatura ambiente estavam com valor acima do recomendado. O pão obteve temperatura mínima de $24,6{ }^{\circ} \mathrm{C}$ e máxima de $25,5^{\circ} \mathrm{C}$ (Tabela 1).

As preparações quentes servidas no almoço, como a isca de carne, obtiveram temperatura mínima de $45,2 \stackrel{\circ}{ } \mathrm{C}$ estando abaixo do recomendado; já o feijão obteve temperatura máxima de $67 \stackrel{\circ}{\circ}$ estando dentro dos padrões recomendados. Ainda com relação ao almoço as preparações servidas em temperatura ambiente como a salada crua de alface e acelga obteve temperatura mínima de $23,5 \stackrel{\circ}{\circ}$, onde elas deveriam ser mantidas sob refrigeração. A mariola servida como sobremesa obteve temperatura máxima de $31,5 \stackrel{\circ}{ } \mathrm{C}$ (Tabela 1).

Para Borges NR et al. (2016), em um restaurante universitário, justificam que provavelmente os fatores que contribuíram para a variação de temperatura foram à ausência de controle da temperatura do armazenamento até a distribuição, equipamentos de aquecimento inadequados e a falta de mecanismos de controle. Alguns fatores que estão relacionados às alterações na temperatura desses alimentos se dão pela ausência de balcão térmico, pela forma como os alimentos se encontram expostos no momento da distribuição e pelo tempo de espera em que as preparações ficam prontas para serem servidas aos alunos.

Em estudo realizado em escolas municipais, foi possível observar que as temperaturas encontradas abaixo do recomendado podem estar relacionadas ao fato de que os alimentos prontos eram mantidos nos mesmos recipientes em que foram produzidos, ficando dispostos sobre mesas e bancadas, à temperatura ambiente $( \pm 25$ $\left.{ }^{\circ} \mathrm{C}\right)$, o que propicia uma maior multiplicação microbiana (MOREIRA SA, et al., 2015).

Os resultados referentes às temperaturas das preparações da UAN II, observou-se que a temperatura da colação e do lanche da tarde em relação às preparações quentes estava abaixo do recomendado, ou seja, inferior 
a $60 \stackrel{\circ}{\circ}$. Alimentos inferiores a essa temperatura podem acarretar patologias em relação à microbiota intestinal, além de surtos de vômitos, diarreia, entre outros sintomas (Tabela 2).

Tabela 2 - Média das temperaturas encontradas nas preparações no início da distribuição da UAN II. Teresina$\mathrm{PI}, 2019$.

\begin{tabular}{|c|c|c|c|c|}
\hline \multirow[b]{2}{*}{ Refeições } & \multicolumn{3}{|c|}{ UAN II } & \multirow[b]{2}{*}{$\begin{array}{l}\text { Recomendada } \\
\left({ }^{\circ} \mathrm{C}\right)\end{array}$} \\
\hline & $\begin{array}{c}\text { Média } \\
\text { Encontrada } \\
\left({ }^{\circ} \mathrm{C}\right)\end{array}$ & $\begin{array}{l}\text { Valor Mínimo } \\
\left({ }^{\circ} \mathrm{C}\right)\end{array}$ & $\begin{array}{c}\text { Valor Máximo } \\
\left({ }^{\circ} \mathrm{C}\right)\end{array}$ & \\
\hline \multicolumn{5}{|l|}{ COLAÇÃO } \\
\hline Preparações Quentes & 34,2 & 51 & 61,8 & $\uparrow 60-65$ \\
\hline Preparações Frias & 8,3 & 9 & 12,8 & Até 10 \\
\hline Preparações Ambientes & 12,4 & 30,9 & 31,2 & $10-21$ \\
\hline \multicolumn{5}{|l|}{ ALMOÇO } \\
\hline Preparações Quentes & 151,3 & 48,7 & 62,7 & $\uparrow 60-65$ \\
\hline Preparações Frias & - & - & - & Até 10 \\
\hline Preparações Ambientes & 21,5 & 25,7 & 28,6 & $10-21$ \\
\hline \multicolumn{5}{|l|}{ LANCHE DA TARDE } \\
\hline Preparações Quentes & 50,7 & - & - & $\uparrow 60-65$ \\
\hline Preparações Frias & 11,3 & 9,6 & 19 & Até 10 \\
\hline Preparações Ambientes & 24 & 26,5 & 32,4 & $10-21$ \\
\hline
\end{tabular}

Fonte: Dados da pesquisa, 2019.

De acordo com a tabela da UAN II pode-se observar que nas preparações quentes servidas na colação como o cachorro-quente obteve temperatura mínima de $51^{\circ} \mathrm{C}$ estando abaixo do recomendado, enquanto o leite apresentou temperatura máxima de $61,8^{\circ} \mathrm{C}$ estando de acordo com o preconizado. Nas preparações frias o suco apresentou temperatura mínima de $9{ }^{\circ} \mathrm{C}$ e a salada de fruta com temperatura máxima de $12,8{ }^{\circ} \mathrm{C}$. Com relação as preparações que eram mantidas em temperatura ambiente, o biscoito de maisena apresentou temperatura mínima de $30,9{ }^{\circ} \mathrm{C}$ e o biscoito doce apresentou temperatura máxima de $31,2{ }^{\circ} \mathrm{C}$. As preparações quentes servidas no almoço, como a bisteca suína, estavam com temperatura abaixo do recomendado com valor mínimo de $48,7 \stackrel{\circ}{\mathrm{C}} \mathrm{e}$ o feijão com valor máximo de $62,7^{\circ} \mathrm{C}$, estando dentro do recomendado. As preparações servidas em temperatura ambiente como a salada crua obteve valor mínimo de $25,7^{\circ} \mathrm{C}$ e a farofa obteve valor máximo de $28,6 \stackrel{\circ}{\circ}$ (Tabela 2).

No lanche da tarde as preparações servidas frias, como o suco, obtiveram temperatura mínima de 9,6 ${ }^{\circ} \mathrm{C}$ e a vitamina de banana com mamão com temperatura máxima de $19 \stackrel{\circ}{\circ} \mathrm{C}$, ou seja, acima do preconizado. As preparações servidas em temperatura ambiente, como o pão, obtiveram temperatura mínima de $26,5{ }^{\circ} \mathrm{C}$ e 0 biscoito de maisena com valor máximo de $32,4 \stackrel{\circ}{ } \mathrm{C}$ (Tabela 2).

Em estudo realizado por Correa VG et al. (2017), em uma Unidade de Alimentação e Nutrição (UAN) universitária que distribui 1000 refeições diárias, afirmam que a média das temperaturas iniciais apresentaram temperaturas acima dos $60^{\circ} \mathrm{C}$, mas que no decorrer da distribuição houve a redução, chegando a $56,1^{\circ} \mathrm{C}$. Assim, esta pode ser uma possível explicação para os resultados encontrados.

As temperaturas encontradas dentro da faixa do recomendado relacionam-se ao fato de serem preparações servidas em um único momento, onde as mesmas são frutas, biscoitos e doces ofertados na UAN escolar. A forma como os alimentos eram distribuídos nesta unidade de alimentação pode ter contribuído para a variação de temperatura devido aos manipuladores proporcionarem as preparações aos pratos antes da distribuição para depois serem distribuídos aos alunos, ficando exposta aquela refeição (Tabela 2). 
Em um Restaurante Universitário na cidade de Salvador/BA, verificou-se que durante a distribuição, a maioria das temperaturas das preparações, como arroz, guarnição e prato principal, apresenta temperatura inferior a $60^{\circ} \mathrm{C}$, no entanto o feijão e a opção apresentam-se em conformidade com o estabelecido pela legislação (RDC 216/2004). Foi observado ainda em relação às saladas crua e cozida, da cadeia fria, que todas estavam acima de $10^{\circ} \mathrm{C}$, ou seja, acima do recomendado pela legislação (CVS 5/2013) (SANTOS LJ, et al., 2013).

\section{CONCLUSÃO}

Com a aplicação de checklist nenhuma das duas UAN's analisadas referentes aos quatro itens alcançaram $100 \%$ de conformidade e que ambas obtiveram falha na adoção de boas práticas, principalmente no item de documentação e registro, ficando evidente o não uso do manual de boas práticas e ausência das POP's. Sabe-se que em relação ao item de equipamentos de proteção individual onde houve baixo percentual de conformidade relacionado ao não uso adequado desses equipamentos, havendo diferença significativa relacionada às duas unidades quando comparadas, devendo haver investimento no treinamento para os funcionários voltado para a Segurança no Trabalho. As temperaturas das preparações ofertadas em ambas às unidades encontraram-se de acordo como determina a portaria CVS 5/2013, dentre elas, as preparações frias e em temperatura ambiente. Já nas preparações servidas quentes, algumas se apresentaram abaixo do padrão recomendado, podendo essas inadequações se relacionarem à ausência de equipamentos como balcão térmico e o momento de sua distribuição. A correção de inadequações encontradas no presente estudo é necessária para as UAN's trabalharem de acordo como a legislação vigente determina, de forma a assegurar a qualidades dos alimentos e permitindo evidenciar de forma simples e prática a importância de checklist como ferramenta fundamental no âmbito da unidade de alimentação e nutrição escolar.

\section{REFERÊNCIAS}

1. BARBOSA ILS. Condições Higiênico-Sanitárias: Um Estudo Retrospectivo em Unidades de Alimentação e Nutrição Hospitalares em Natal-RN. Dissertação (Trabalho de Conclusão de Curso em Nutrição) - Centro de Ciências da Saúde. Universidade Federal do Rio Grande do Norte, Natal, 2017. 67 p.

2. BORGES NR, et al. Avaliação do binômio tempo-temperatura das refeições de um restaurante na cidade de Palmas - Tocantins. Rev Desafios. 2016; 3(2):90-98.

3. CORREA VG, et al. Monitoramento do binômio tempo e temperatura nos processos de produção de alimentos em um restaurante universitário. Rev Brazilian Journal of Food Research. 2017; 8(2):46-56.

4. GOMES MFFA, et al. Alimento Seguro - Escola Saudável. Rev Extilfal. 2015; 8(6):19-28.

5. GOMES NAAA, et al. Aspectos higiênico-sanitários no processo produtivo dos alimentos em escolas públicas do Estado de Goiás, Brasil. Rev de Nutrição. 2012; 25(4):473-485.

6. GOMES RNS, et al. Adequação higiênicos sanitária de unidades de alimentação e nutrição de escolas públicas em município do maranhão. Rev Higiene Alimentar. 2016; 30(260): 38-42.

7. LOPES ACC, et al. Avaliação das Boas Práticas em unidades de alimentação e nutrição de escolas públicas do município de Bayeux, PB, Brasil. Rev Ciência \& Saúde Coletiva. 2015; 20(7):2267-2275.

8. MEDEIROS MGGA, et al. Percepção Sobre a Higiene dos Manipuladores de Alimentos e Perfil Microbiológico em Restaurante Universitário. Rev Ciência \& Saúde Coletiva. 2017; 22(2):383-392.

9. MELO VL, et al. Implicações Dos Equipamentos de Proteção Individual Na Psicodinâmica do Trabalho. Rev de Enfermagem UFPE. 2014;8(6):1617-1627.

10. MOREIRA SA, et al. Temperatura de alimentos durante a distribuição em unidades de alimentação e nutrição escolares de ItaquiRS. 2015; 7(2):63-70.

11. NETO AB, et al. Conhecimento Antes e Depois de Um Treinamento de Boas Práticas em Serviços de Alimentação para Manipuladores e Responsáveis Técnicos. Rev Nutrição em Pauta. 2015; 23(131):36-41.

12. NUNES CNM, et al. Implantação dos procedimentos operacionais padronizados (POPs) de higienização e desinfecção dos equipamentos e utensílios de uma unidade de alimentação e nutrição hospitalar. Rev. Simbio-Logias. 2014; 7(10):34-48.

13. PEDERSSETTI MT, HAUTRIVE TP. Condições higiênico-sanitárias de Unidades de Alimentação e Nutrição Hospitalares da Região Oeste de Santa Catarina. Rev Segurança Alimentar e Nutricional. 2016; 23(1):849-858.

14. POHREN NF, et al. Avaliação da estrutura física de uma unidade de alimentação e nutrição. Rev Univap. 2014; 20(36): 17-23.

15. RAMOS AE, et al. Avaliação das condições higiênico-sanitárias das cozinhas de escolas públicas de um município do semiárido nordestino. Rev. Interd. Ciências e Saúde. 2015; 2(3):64-73.

16. RASQUINHA BS, et al. Avaliação das condições higiênico-sanitárias em unidades de alimentação escolar da rede municipal de um município do vale do rio pardo, rio grande do sul. Rev Caderno pedagógico. 2017; 14(2):45-55.

17. RIBEIRO ESS. Condições Higiênico-Sanitárias de Uma Unidade de Alimentação e nutrição Hospitalar: Manipuladores de Alimentos em Foco. Dissertação (Trabalho de Conclusão de Curso em Nutrição) - Centro de Ciências da Saúde Departamento de Nutrição, Universidade Federal do Rio Grande do Norte, Natal, 2017. 85 p.

18. SANTOS LJ, et al. Tempo e temperatura da alimentação transportada destinada ao restaurante universitário na cidade de Salvador - BA. Rev Saúde e Pesquisa. 2013; 7(9): 23-31. 
19. SILVA LM, et al. Avaliação das condições higiênicas de escolas de ensino infantil e fundamental por meio da aplicação de listas de verificação. Rev Segurança Alimentar e Nutricional. 2016; 23(1):837-848.

20. TRASEL J, JUNIOR SBJ. Implantação de Boas Práticas de Fabricação em empresa de Chocolates Artesanais em Arroio do Meio. Lajeado: Centro Universitário Univates, 2014. Graduação Técnico em Química.

21. WIETHOLTER MJ, FASSINA P. Temperaturas de armazenamento e distribuição dos alimentos. Ver Segurança Alimentar e Nutricional. 2017; 24(1):17-25

22. ZANETIN PM, FATEL ECS. Avaliação da ergonomia e do Uso de Equipamentos de Proteção Individual em Unidades Produtoras de Refeições. Rev RASBRAN - Revista da Associação Brasileira de Nutrição. 2017; 8(1):90-100. 